\title{
Stagnation point flow over a stretching/shrinking cylinder with prescribed surface heat flux
}

\begin{abstract}
The steady stagnation-point flow towards a horizontal linearly stretching/shrinking cylinder immersed in an incompressible viscous fluid with prescribed surface heat flux is investigated. The governing partial differential equations in cylindrical form are transformed into ordinary differential equations by similarity transformations. The transformed equations are solved numerically by using the shooting method. Results for the skin friction coefficient, local Nusselt number, velocity profiles and temperature profiles are presented for different values of the governing parameters. Effects of the curvature parameter, stretching/shrinking parameter and Prandtl number on the flow and heat transfer characteristics are discussed. The study indicate that the solutions for a shrinking cylinder are non-unique. It is observed that the surface shear stress and heat transfer rate at the surface increase as the curvature parameter increases.
\end{abstract}

Keyword: Boundary layer; Dual solutions; Heat transfer; Stagnation point flow; Stretching/shrinking cylinder 